\title{
The Poincaré Section Method for Beam Element Transfer Maps
}

\author{
Ravi Jagasia, Martin Berz and Ben Loseth
}

Department of Physics and Astronomy, Michigan State University, East Lansing, Michigan, USA

The computation of particle trajectories in particle optical elements is a task that has been continuously improved upon since the advent of accelerators, electron microscopes, spectrographs, and other particle optical systems. In the past transfer maps for the simplest of elements would be computed by hand to a few orders, codes such as COSY INFINITY now compute numerous elements to arbitrary order [1,8]. The next logical step is in the direction of the computation of an element of an arbitrary electromagnetic field to an arbitrary order. The demand for this step is imminent in the requirements for higher precision and higher orders in various types of particle optical systems, including the simulation of the storage ring of the cooler synchrotron in Jülich for electric dipole moment (EDM) searches [2]. In addition, conventional map methods are not natively suitable for the treatment of the radio frequency Wien filter which is an essential piece of equipment for studying the EDM at this facility in the frozen-spin method $[3,4]$.

Convention and convenience in the beam physics community ask that for a given apparatus we are looking for a map that takes the initial state of particles as they enter the device to that of a final state as they exit. However, in general, we aren't looking to provide a relationship between the initial state of particles and their final states at a given time; rather, we are looking for the relationship between the two at two different planes in space. Namely, we are looking for a map that relates the state of particles $z_{i}$ on an initial plane $s_{0}=0$ to that of the state $z_{f}$ on the final plane $s_{f}$ as they travel through a specified electromagnetic field. This map can be obtained to arbitrary order within the framework of the code COSY INFINITY through the use of differential algebras (DA) [9].

Often in dynamical systems, we ask for a mathematical construct called a Poincaré section in which we keep track of locations on a given plane that an integrated orbit passes through [5]. This generally makes it easier for one of the requirements of performing a Poincare section in a differential algebraic framework. Namely, a crucial step in generating a Poincaré section is that of map inversion. Map inversion generally requires that we have a map that is origin preserving, i.e. $M(0)=0$. For a singular beam element this condition is obviously not met automatically, but the pure translation of the origin can be subtracted out for this purpose. This is natural in the case of examining all but bending elements, but they can also be included in this treatment by simple rotations.

The map that we wish to construct is the Poincaré section that takes a set of initial conditions that correspond to the structure expected by usual map methods, simply put: a plane perpendicular to the optical axis. For convenience, we look to provide our Poincare section in the particle optical coordinates. This makes the result compatible for comparison and usability of traditional map methods. These coordinates have the benefit of being useful to experimentalists and for comparison to standard beam techniques and tools. The transformation from local geometrical coordinates are

$$
\begin{array}{rlrc}
x, & a & & p_{x} / p_{0}, \\
y, & b & = & p_{y} / p_{0}, \\
l=-\gamma_{0} v_{0}\left(t-t_{0}\right) /\left(\gamma_{0}+1\right), & \delta_{k} & = & \left(E_{k}-E_{k 0}\right) / E_{k 0} .
\end{array}
$$


The relativistic relationships $\gamma=1 / \sqrt{1-v^{2} / c^{2}}, E=\sqrt{p^{2} c^{2}+m^{2} c^{4}}$ and $E_{k}=E-m c^{2}$ are used with the note that $m$ refers to the rest mass, and we do not use the relativistic mass to avoid confusion. In these coordinates quantities are provided relative to a reference particle that is identified by the subscript 0 . The map description is defined relative to the reference particle which is defined to be at the origin at the beginning of the integration. The remainder of the map can be visualized to exist as a Poincaré section with an added translation along the coordinate of the particle trajectory $s$.

The usual choice for map methods is to perform integration in the particle optical coordinates, in this the usual Lorentz equations take the form of the so called particle optical equations of motion [1]:

$$
\begin{gathered}
\frac{d x}{d s}=(1+h x) \frac{a}{\sqrt{\left(p / p_{0}\right)^{2}-a^{2}-b^{2}}}, \quad \frac{d y}{d s}=(1+h x) \frac{b}{\sqrt{\left(p / p_{0}\right)^{2}-a^{2}-b^{2}}}, \\
\frac{d a}{d s}=h \cdot \sqrt{\left(p / p_{0}\right)^{2}-a^{2}-b^{2}}+\frac{e}{p_{0}}\left[\frac{1}{\dot{s}} E_{x}+\frac{d y}{d s} B_{s}-(1+h x) B_{y}\right], \\
\frac{d b}{d s}=\frac{e}{p_{0}}\left[\frac{1}{\dot{s}} E_{y}-\frac{d y}{d s} B_{s}+(1+h x) B_{x}\right] .
\end{gathered}
$$

This provides for a straightforward integration between the effective field boundaries; however, the inclusion of time varying fields is not easy in these coordinates since we do not know the reference particle trajectory a priori. Instead, we opt for Cartesian coordinates and integrate in the familiar Lorentz equations suitably scaled for beam physics computations to minimize floating point rounding errors by choosing appropriate units (also: $\tau=t c$ and $\vec{\beta}=\vec{v} / c$ ):

$$
\frac{d \vec{x}}{d \tau}=\vec{\beta}=\frac{\vec{p}}{\sqrt{m^{2} c^{2}+p^{2}}}, \quad \quad \frac{d \vec{p}}{d \tau}=\frac{q}{c} \vec{E}+\frac{q \vec{p}}{\sqrt{m^{2} c^{2}+p^{2}}} \times \vec{B} .
$$

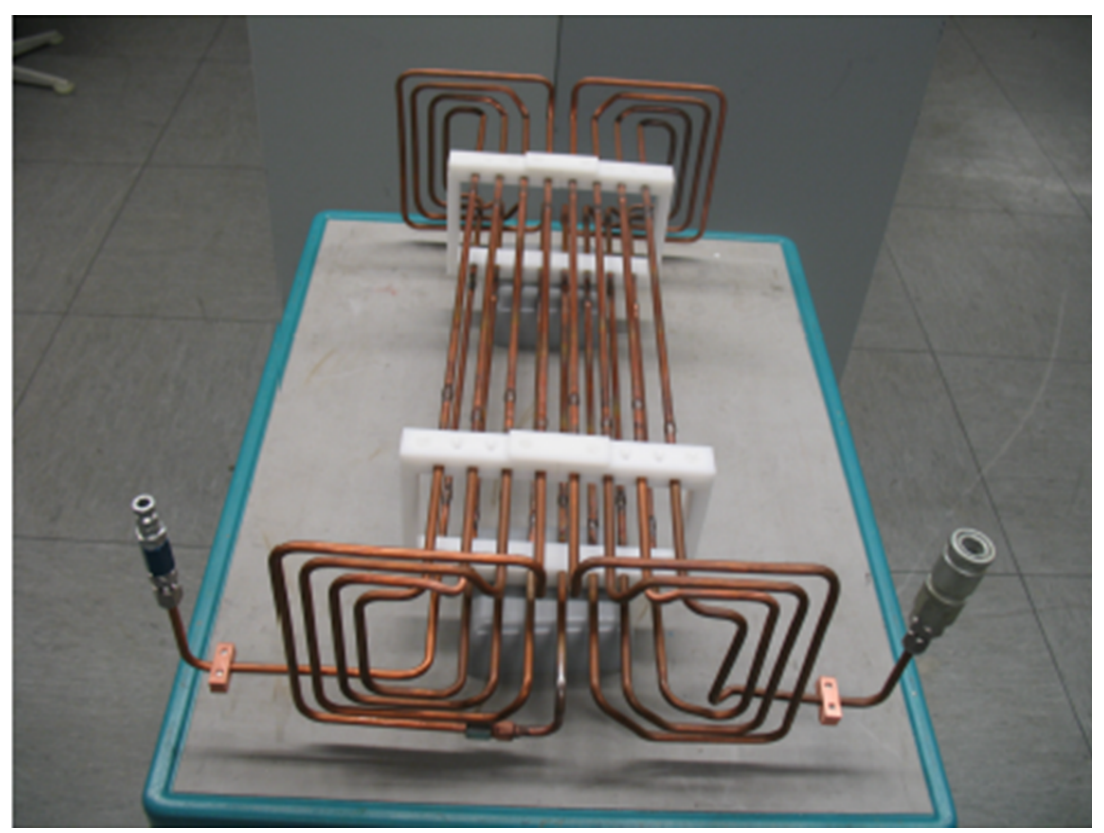

Figure 1. Wien filter wire prototype structure without inclusion of plate electrostatic deflector [2]. 


\begin{tabular}{|c|c|c|c|}
\hline$\vec{x}$ in $\mathrm{m}$ & $\vec{p}$ in $\mathrm{MeV} / c$ & $q$ in $e$ & $m$ in $\mathrm{MeV} / c^{2}$ \\
\hline$\vec{E}$ in $\mathrm{MV} / \mathrm{m}$ & $\vec{B}$ in $\mathrm{T}$ & $c$ in $\mathrm{Mm} / \mathrm{s}$ & \\
\hline
\end{tabular}

In order to maintain the same type of initial conditions, the different coordinate system means that our initial conditions are less straightforward, but they are designed to be that which corresponds to particles with a given kinetic energy, mass and charge. Treating $p_{x}$ and $p_{y}$ as free parameters, we specify $p_{z}=$ $\sqrt{p^{2}-\left(p_{x}^{2}+p_{y}^{2}\right)}$ in which we have the usual $p c=\sqrt{\left(E_{k}+m c^{2}\right)^{2}-m^{2} c^{4}}$.

Since we are performing a flow integration it is necessary to prescribe the initial conditions in terms of flows. For position we require expansions in the $x$ and $y$ coordinates where we maintain everything in units of meters. For readability the flow expansions are done such that $x=x_{0}+d x$ but we note that for such a large expansion ( $d x$ is a computational value assumed to vary from -1 to 1 ) we will need to use appropriate norms during integration since we are only concerned with a smaller region about the reference trajectory. The $z$ coordinate is specified as a constant since we are looking at a planar object. Noting that $E_{k}=E_{k 0}\left(1+\delta_{k}\right)$ we also treat $\delta_{k}$ as a free parameter and can use these expressions to generate differential algebraic vectors that reflect the quantities up to an arbitrary desired order.

We must then perform a shooting method to find the time $t_{r}$ when the reference particle intersects with the plane corresponding to the chosen final conditions. This is done by a traditional adaptive Runge-Kutta integrator of orders 7 and 8. For the purposes of computing a Poincaré section, it is required that we have a time expansion of the flow around the plane for which we wish to generate a map, this is because in the Lorentz equations we integrate with respect to the independent variable time and all particles do not intersect our final plane simultaneously. In this, a flow integrator is needed in order to compute the time of flight for the entire section. For generating these time expansions we iterate the Picard-Lindelöf operator [1] to create a flow expansion in time such that for each initial condition there exists a time for which the particle is on the chosen final condition plane:

$$
\vec{x}=A(\vec{x})=\vec{x}_{i}+\int_{t_{i}}^{t} \vec{f}\left(\vec{x}, t^{\prime}\right) d t^{\prime} .
$$

Then we perform a differential algebra based constraint satisfaction algorithm for which we require our particles are on our final condition plane which allows us to obtain the time of flight for all particles described by this flow. Inserting this into our flow expansion provides us with a Cartesian coordinate map as a function of their initial coordinates. The transformation from here to particle optical coordinates is straightforward.

The computation of maps has previously been done for specific known systems such as magnetic and electric dipoles, quadrupoles, and higher order components up to what is generally useful to work out by hand and write into code. The Poincaré section method is a more general solution, but must lead to the same results for standard particle optical elements. The primary verification of the algorithm is done via the comparison to standard accelerator elements as implemented in COSY INFINITY. A number of cases are examined, each with high order components. The first case of a cross comparison is a magnetic quadrupole in which we find that the resulting maps agree to high precision of the order $10^{-12}$ in the map itself without considering the coordinate scaling assumed for high order components. 


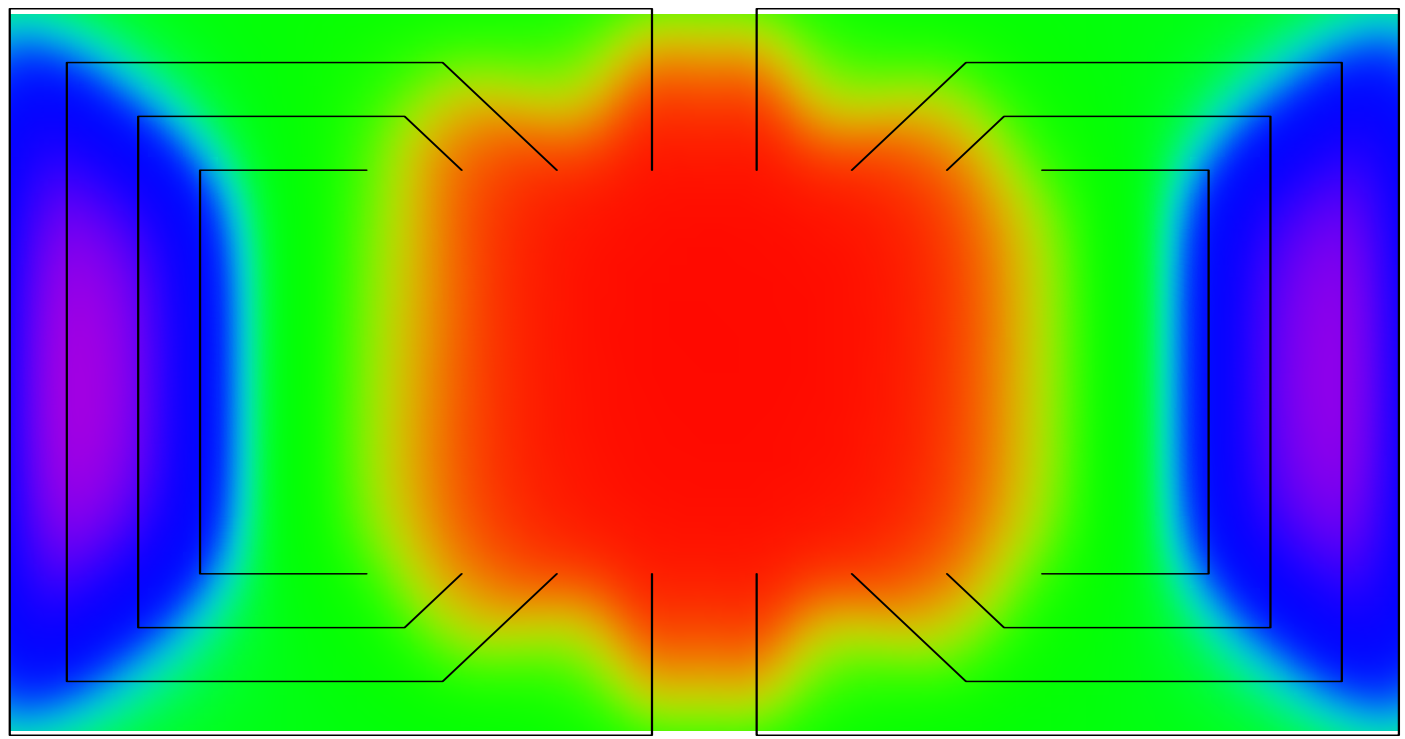

Figure 2. Differential algebra based Biot-Savart computation for the magnetic field with wire pieces superimposed in the $x-y$ plane $20 \mathrm{~mm}$ before entrance to the wire pieces looking down the $z$ axis through the front of Figure 1 through the cavity. Shown is $B_{x}$ with the total dimensions $146 \times 80 \mathrm{~mm}$, range from $0.227 \mathrm{mT}$ (purple) to $0 \mathrm{mT}$ (green) to $-0.150 \mathrm{mT}$ (red).

The second case is that of an ideal Wien filter, a velocity filter corresponding to constant $\vec{B}$ and $\vec{E}$ fields such that particles of the chosen specific velocity traverse the element without deflection. In this case, we also see high precision agreement with the equivalent COSY INFINITY beam physics package function.

For the purposes of considering a more realistic example that includes fringe fields which have usually been modeled via Enge functions [10], we examine the equations corresponding to a bar magnet [7] in which $X_{i}=x-x_{i}, Y_{ \pm}=y_{0} \pm y, Z_{i}=z-z_{i}$, and $R_{i j}^{ \pm}=\sqrt{X_{i}^{2}+Y_{ \pm}^{2}+Z_{j}^{2}}$ :

$$
\begin{aligned}
& B_{x}(x, y, z)=\frac{B_{0}}{4 \pi} \sum_{i, j=1}^{2}(-1)^{i+j}\left[\ln \left(\frac{Z_{j}+R_{i j}^{-}}{Z_{j}+R_{i j}^{+}}\right)\right], \\
& B_{y}(x, y, z)=\frac{B_{0}}{4 \pi} \sum_{i, j=1}^{2}(-1)^{i+j}\left[\arctan \left(\frac{X_{i} \cdot Z_{j}}{Y_{+} \cdot R_{i j}^{+}}\right)+\arctan \left(\frac{X_{i} \cdot Z_{j}}{Y_{-} \cdot R_{i j}^{-}}\right)\right], \\
& B_{z}(x, y, z)=\frac{B_{0}}{4 \pi} \sum_{i, j=1}^{2}(-1)^{i+j}\left[\ln \left(\frac{X_{i}+R_{i j}^{-}}{X_{i}+R_{i j}^{+}}\right)\right] .
\end{aligned}
$$

By taking a rotation about the $z$ axis and scaling appropriately this bar field can be used to create a Wien filter by considering it as an electric field. By construction with scaling to compensate forces of parts of Lorentz force law, the combination of these two bar fields exactly cancel on the $z$ axis for a given velocity, and the reference particle follows the straight line orbit that is expected of an ideal Wien filter both in the interior and in the fringe region.

The magnetic fields are provided by direct computation using the Biot-Savart law from wire pieces that 
have been bent into the shape shown in Figure 1. The shape was then measured and the data of the wire pieces were provided to us by Denis Zyuzin. The COSY INFINITY code to generate a differential algebraic expansion of the field given a set of currents was then used since the computation of the Poincaré maps requires expansions of the field at every integration point. A contour plot of the magnetic field $B_{x}$ of this with superimposed wire pieces as seen along the beam axis in the $z$ direction is shown in Figure 2. The code used to generate a differential algebraic expansion of the field is done in two situations: first, approximating all the wire pieces as straight line segments, then second, having all wires shortened and circular segments used for the corners of a specific radius.

The maximum current amplitude $I=10$ A which according to the JEDI COSY Proposal [2] should provide an integrated field along the beam axis of $0.33 \mathrm{~T} \mathrm{~mm}$, with a maximum field of $0.59 \mathrm{mT}$ on the $x-z$ beam plane. In the envisioned case of deuterons at $970 \mathrm{MeV} / \mathrm{c}$, the voltage on the capacitor that compensates the influence of the magnetic field is $3936 \mathrm{~V}$, corresponding to an $E_{y}$ of $75.840 \mathrm{kV} / \mathrm{m}$. Unfortunately, while the shape of the magnetic field is well specified, our description of our compensating electric dipole field is less accurate. In lieu of this, we use our simulated bar field for the electric field. In a perfect Wien filter our reference particle trajectory is a straight line; however, for real fields this is not possible. By using our electric bar field we can change the field strength and adjust length of the electric bar field which give handles on midfield maximum strength and the integrated field strength which are parameters that are useful for matching the goal of having a minimal exit angle of the reference orbit considering an entrance angle of zero. Since we are optimizing for exit angle with only two parameters, we do end up with constant offsets in the maps.

Unfortunately, this wire model does not fully depict the system as there will be iron blocks used to flatten the field. This requires a different method to compute the field. One possibility is that of performing a much more complicated field computation that includes the magnetic permeability of the iron. However, this would be a fairly specific computation. For more general computations of fields, we instead consider the Helmholtz equations for a field given by $[6,7]$ :

$$
\vec{F}=-\vec{\nabla} \phi+\vec{\nabla} \times \vec{A}
$$

in which we have a given field decomposed into two parts, described by $\phi$, the irrotational or curl free part, and $\vec{A}$, the solenoidal or divergence free part. The individual parts are given by:

$$
\begin{aligned}
\phi(\vec{r}) & =\frac{1}{4 \pi} \int_{V} \frac{\vec{\nabla}^{\prime} \cdot \vec{F}\left(\vec{r}^{\prime}\right)}{\left|\vec{r}-\vec{r}^{\prime}\right|} d V^{\prime}-\frac{1}{4 \pi} \int_{S} \frac{\vec{F}\left(\vec{r}^{\prime}\right) \cdot d \vec{S}^{\prime}}{\left|\vec{r}-\vec{r}^{\prime}\right|} \\
\vec{A}(\vec{r}) & =\frac{1}{4 \pi} \int_{V} \frac{\vec{\nabla}^{\prime} \times \vec{F}\left(\vec{r}^{\prime}\right)}{\left|\vec{r}-\vec{r}^{\prime}\right|} d V^{\prime}+\frac{1}{4 \pi} \int_{S} \frac{\vec{F}\left(\vec{r}^{\prime}\right) \times d \vec{S}^{\prime}}{\left|\vec{r}-\vec{r}^{\prime}\right|}
\end{aligned}
$$

For Maxwell's equations in free space for time-independent fields the equations reduce to purely surface integrals:

$$
\begin{array}{cl}
\nabla \cdot \vec{B}=0, & \nabla \times \vec{B}=\mu_{0} \vec{J}+\mu_{0} \varepsilon_{0} \frac{\partial \vec{E}}{\partial t}=0, \\
\phi(\vec{r})=-\frac{1}{4 \pi} \int_{S} \frac{\vec{F}\left(\vec{r}^{\prime}\right) \cdot d \vec{S}^{\prime}}{\left|\vec{r}-\vec{r}^{\prime}\right|}, & \vec{A}(\vec{r})=\frac{1}{4 \pi} \int_{S} \frac{\vec{F}\left(\vec{r}^{\prime}\right) \times d \vec{S}^{\prime}}{\left|\vec{r}-\vec{r}^{\prime}\right|} .
\end{array}
$$


These surface integrals, evaluated in differential algebra, easily provide us with the fields on the interior of a surface of measured data. This data of course has to be cast into the form of a Taylor expandable form, but this is done using Gaussian interpolation which provides us with expansions of arbitrary order. In this fashion, we can take measured data with a specific surface geometry, and construct the fields needed for the flow integration in order to generate the map for an arbitrary field distribution.

This differential algebraic algorithm allows us to generate maps for both prescribed fields and for surface data through the Helmholtz equation which provides averaging as compared to midplane expansion methods. This general mechanism for the generation of maps for accelerator elements allows for inclusion of the entire field rather than a decomposition into fringe fields and multipole expansions. In this we have shown that the method is readily applicable to the analysis of increasingly more complex systems such as the Wien filter designed to be used in the storage ring for EDM searches [11].

\section{References:}

[1] M Berz, "Modern map methods in particle beam physics", (Academic Press, San Diego, 1999).

[2] A Lehrach et al, "Search for electric dipole moments at COSY", JEDI Collaboration Proposal, (Forschungszentrum Jülich, Jülich, 2014).

[3] F Rathmann et al, Physics of Particles and Nuclei 45 (2014), p. 229.

[4] S R Mane, Nuclear Instruments and Methods A 596 (2008), p. 288.

[5] J Grote et al, Nuclear Instruments and Methods A 558 (2006), p. 106.

[6] S Manikonda and M Berz, International Journal of Pure and Applied Mathematics 23 (2005), p. 365.

[7] S Manikonda, Nuclear Instruments and Methods A 555 (2006), p. 175.

[8] M Berz, Nuclear Instruments and Methods A 298 (1990), p. 473.

[9] M Berz, Particle Accelerators 24 (1989), p. 109.

[10] M Berz, Nuclear Instruments and Methods A 363 (1995), p. 100.

[11] We thank Marcel Rosenthal, Denis Zyuzin and Andreas Lehrach at Forschungszentrum Jülich for the photo and data of the Wien filter, and Kyoko Makino at Michigan State University for useful comments. We are grateful for financial support from the US Department of Energy under grant DE-FG0208ER41546. 